\title{
Demonstrating the Spread of Bacterial Infection Within a Hospital Setting through Simulations and Role-Play
}

\author{
Hannah Lathrop, Lindsey Hull, Lexi Green, Susan Wright, Sarah E. Ruffell
}

\author{
University of Pittsburgh at Bradford, Division of Biological and Health Sciences, 300 Campus \\ Dr, Bradford PA 16701 USA \\ (Imh141@pitt.edu;lag135@pitt.edu; hj121@pitt.edu; sjw80@pitt.edu;ruffell@pitt.edu)
}

\begin{abstract}
The purpose of this lab is to showcase how infections and bacteria are spread throughout the hospital from patient to healthcare personnel. Students were assigned to specific roles, such as patient, nurse, physician, family member, lab technician and hospital liaison. Through the use of simulations and role-playing students worked as a team to determine the patient's diagnosis. This activity was designed for nursing students, and could be completed in group of 4 over a 1-hour period.
\end{abstract}

Keywords: Microorganisms, UV light, UV powder, hospital personnel

\section{Introduction}

Role-playing exercises allow students to practice the roles of a professional or participate in a specific situation (Northern Illinois University 2019). During this exercise, students performed tasks from the perspective of a health care professional, such as a doctor or nurse. The tasks the students performed were vital checks, using proper safety protocols, and reading lab results. A roleplaying exercise, such as this one, is beneficial to nursing students because it allows them to learn new skills, while reaffirming current knowledge.

Situated learning occurs when students can take part in an immersive experience based on real-life situations (Northern Illinois University 2019). This style of learning encourages students to work as a team and participate in tasks that require critical thinking (Northern Illinois University 2019). This exercise prepares these students by educating them about hospital-acquired infections (HAIs) and the precautions taken to prevent these infections from spreading. Hospital-acquired infections or nosocomial infections are afflictions that patients acquire while receiving treatment from a medical facility (Massachusetts Health \& Hospital Association 2019). These illnesses are spread through two forms of contact: direct and indirect (Drexler 2010; Massachusetts Health \& Hospital Association). Indirect contact is the most common way pathogens spread in a medical setting (Drexler 2010). Indirect contact occurs through the transmission of microbes between shared surfaces, such as doorknobs, patient rooms, and the droplets created through sneezing and coughing (Drexler 2010). The five most common microbes that are found within a medical setting include Acinetobacter baumannii, Clostridium difficile, Escherichia coli, Methicillin-resistant Staphylococcus aureus, and Pseudomonas aeruginosa (Becker's Healthcare 2014). These pathogens are found to infect one out of twenty people within a medical setting each day (Becker's Healthcare 2014). However, hospitals and nursing programs strive to be more diligent to reduce the spread of these ailments.

Hospitals and nursing programs implement guidelines on how healthcare professions can prevent the spread of hospital-acquired infections. The guidelines are divided into standard and specific precautions (Mehta et al., 2014). Standard precautions, such as using proper personal safety equipment and practicing proper handwashing, are to be used daily; however, they are effective in decreasing the spread of HAIs (Mehta et al., 2014). Another way to prevent HAIs is to inform patients and their families of these illnesses and their symptoms. Medical facilities should also provide patients and their families with personal safety equipment, like face shields and handwashing stations, to ensure the safety of all people in the facility (Mehta et.al., 2014).

Students participated proper hospital sanitation techniques through a role-playing situated learning activity. At the start of this experiment, the patient in each group will be given UV powder to rub on their hands that will transfer to the other members in the group when they come into contact with the patient. Next, the groups will perform their assigned tasks according to the role card they have received, and together, they will determine their 
patients' diagnosis based on their signs and symptoms. At the conclusion of this activity, a reveal will take place that will show the students how bacteria (UV powder) were transferred from person to person. To achieve this reveal, a UV light will be used. The activity will take approximately 30-40 minutes from start to finish. This activity emphasizes proper hospital protocol through a variety of pedagogical tools. 


\section{Student Outline}

\section{Objectives}

- Apply knowledge of hospital-acquired infections

- Describe and understand how microorganisms are easily spread

- Execute experiment for better understanding on the topic

\section{Background Information}

Nosocomial infections or hospital-acquired infections are ailments that patients obtain while receiving medical treatment (Massachusetts Health \& Hospital Association 2019). These infections can be spread through the air, or from direct or indirect contact with another person. Airborne infections are spread by someone sneezing or coughing, while indirect infections are spread through a person encountering an infected surface such as a doorknob. All hospitalized patients are at risk of obtaining a nosocomial infection (World Health Organization 2002; Massachusetts Health \& Hospital Association 2019). The patients who have a high risk of developing a nosocomial infection include the elderly, children, those with compromised immune systems, patients with indwelling catheters, patients who overuse antibiotics or the healthcare professionals that negate to wash their hands (Centers for Disease Control 2015). About 1.7 million infections and 99,000 deaths associated with hospitalacquired infections occur every year in American hospitals (Massachusetts Health \& Hospital Association 2019). The most successful way to prevent these infections is for healthcare professionals to wash their hands before entering and leaving a patient's room.

In this laboratory exercise, we will demonstrate the importance of handwashing by demonstrating the spread of bacteria through direct transfer. During this activity, you will be working in small groups and preforming tasks based on various roles within a hospital setting. Following the tasks outlined on your role card, a diagnosis will be determined through the use of an assessments and your diagnostic skills.

\section{Methods and Data Collection}

1. The instructor will assign groups of four. The groups of 4 will include a nurse, physician, family member, and patient. Two students within the class will not be assigned to a group and will be the liaison and the lab technician who will float from group to group performing their specific tasks. All students will receive a card with the necessary duties/tasks that he/she needs to perform during the exercise.

2. Additionally, the assigned patient in each group will be given UV powder to rub on their hands that will transfer to the other members in the group when they come into contact with the patient.

2. The scenario for each group is:

A 35-year-old female/male is admitted to the floor complaining of watery diarrhea for two consecutive days and just finished a 10-day course of antibiotics 5-days ago to treat community-acquired pneumonia.

Her/his abdomen is soft with hyperactive bowel sounds, and she/he is reporting abdominal cramping pain

of a 3 on a scale of 1-10 and complains of nausea. Her/his lungs are clear, and she/he has prolonged

tenting when checking skin turgor. Record the patients vitals on the chart.

Temperature: $101.4^{\circ} \mathrm{F}$

Pulse: 102

Respirations: 22

Blood Pressure: 104/62

O2 Sat: $98 \%$

(Centers for Disease Control 2015; Centers for Disease Control 2018)

3. The assigned doctor and nurse will fill in the patient's vital signs in Figure 1. Vitals Signs Flow Sheet. A Vital Signs Flow Sheet with sample data can be found in Appendix A. 


\section{(1) Vital Signs Flow Sheet}

PATIENT'S NAME:

\begin{tabular}{|c|l|l|c|l|l|l|c|}
\hline Date: & Temperature: & Pulse: & $\begin{array}{c}\text { Blood } \\
\text { Pressure: }\end{array}$ & Respirations: & $\mathrm{O}_{2}$ Sat: & Pain: & Initials \\
\hline & & & & & & & \\
\hline & & & & & & & \\
\hline & & & & & & & \\
\hline
\end{tabular}

Other Symptoms:

Figure 1. Vital signs flow sheet for nurse and doctor to fill out.

4. Although each student has a different role, everyone must work together to determine the final diagnosis of the patient. The goal of the exercise is to work together to determine the diagnosis of the patient by performing exams and techniques that would happen in a hospital setting. By the end of the exercise all the groups will have to determine the diagnosis of their patient and will share it with the class.

5. At the conclusion of the diagnosis part of the activity, a reveal will take place that will show students how bacteria in the simulation using UV powder were transferred from person to person. To achieve this reveal, a UV light will be used. See Appendix B to view photographs of bacterial transfer as exemplified by UV powder.

6. The activity will take approximately 30-40 minutes from start to finish. This activity emphasizes proper hospital protocol through a variety of pedagogical tools. 


\section{Materials}

The supplies needed are as follows:

- UV Powder: for this exercise we specifically used Glo Germ powder, which comes in three different colors (White, Yellow, Orange). We used the white powder. All powders can be purchased from http://www.glogerm.com/mm5/merchant.mvc for an estimated cost of $\$ 18.00$ USD. Glo Germ powder is made from $100 \%$ Melamine Resin (plastic) (Glo Germ 2014).

- Cosmetic Sponges: Use these to apply the UV powder to the patient's hand and/or surfaces within the classroom can be purchased from Amazon for an estimated cost starting at $\$ 1.00$ USD.

- UV Flashlight: Used for the reveal of the spread of microbes at the end of the lab. UV flashlights can be purchased from Amazon for an estimated cost of \$8.00 USD.

- $\quad$ Role Cards and Patient Information: which can be found in Part C and E of the student outline

- Vital Signs Chart: To be filled out by students with the roles of doctor and nurse. Also used to notate the symptoms of the patient being examined and can be found in Part E.

- Brown Paper Bag: Used to resemble the stool sample and blood work that has been done by the lab technician

\section{Optional Materials}

These materials can be used by the students while performing the diagnostic procedures needed to get the patients information.

- Blood Pressure Gauge: Used by the nurse or doctor to take the patient's blood pressure

- Pulse Oximeters: Used by nurse or doctor to measure the level of oxygen saturation within the patient's blood.

- Medical Thermometer and Probe Covers: Used by nurse or doctor to take the patient's temperature

\section{Notes for the Instructor}

Prior to this lab instructors may consider giving a lecture explaining the mechanisms that spread infections. Please provide students with the study guide for this topic which can be found in the appendix.

After step 1 in the student handout, the instructor will now call the patients out into the hallway. These students will be given information about their symptoms, and the UV powder will be applied to one of their hands. Be sure to explain to the patients that they are not to provide any information about their symptoms unless their vitals are taken or a specific question about a symptom is asked. After students complete step 4 and the diagnosis is determined. The UV powder is revealed and students can use the UV lights to determine where the UV travelled on each person. The instructor will then lead the students in a post lab discussion about the importance of this exercise. Refer to Appendix for handouts, keys and images illustrating procedure.

For this lab many modifications can be made to incorporate this lab into courses other than those for students pursuing a degree in health sciences. Please refer to the following subsections to make modifications as needed.

\section{General Modifications for this Exercise}

There are some modifications that can be made to this exercise to accept changes such as group sizing and the use of roles. To accommodate smaller groups, the roles of family member and liaison may be removed.

This exercise can also be used multiple times through the addition of a second patient. Adding a second patient will allow students to have a visual of patient-topatient contact and will also allow the exercise to be more challenging.

Instructors may also consider putting the glow germ powder on the medical equipment being used along with other surfaces throughout the room. Doing this will allow students to have a visual of why it is important to use proper handwashing. Along with expressing the importance of cleaning all surfaces and equipment thoroughly.

\section{Modifications for Nursing Courses}

This exercise can be used as a diagnostic tool for nursing students to check if they are using medical equipment properly, along with maintaining a proper bedside manner. This will help prepare students for realworld events within a medical setting.

\section{Modifications for Biology Courses}

This exercise can be used as is, but we suggest taking some time to do a mini lecture on medical terminology and proper vital collection. Also provide students with the medical terminology quick guide and guide to checking patient's vitals which can be found within the appendix.

This exercise can be changed to help teach students about why it is important to practice proper safety protocols within a biology lab setting. To display this, you can use the UV powder to represent a toxic material that may be used within a laboratory setting. Place the UV powder on surfaces and tools around the room. Then allow the students to practice using the different tools while using the proper safety gear. Then use the UV light to expose the 
toxic substance. This lab modification can show biology students the importance of using the proper safety protocols within a laboratory setting.

\section{Modifications for Non-Major Biology Courses}

To use this lab within a non-majors biology course, instructors may consider changing the scenario. This lab modification can show students the importance of using the proper hygiene within a dorm room setting. Place the UV powder on items such as dishware, clothing, electronics, linens, doorknobs, and other surfaces within the classroom. Give each student a task like typing on a laptop. After they have completed their individual task and interacted with another student, perform the UV light reveal. Then hold a facilitated discussion on why it is important to practice good hygiene to keep one from getting sick during the school year.

\section{Cited References}

Clostroiodes difficile Infection. c2015. Atlanta (GA): Centers for Disease Control - Healthcare Acquired Infections. [updated 2015 Feb. 25; cited 2019 Sept. 16]. https://www.cdc.gov/hai/organisms/cdiff/cdiff_i nfect.html.

Drexler, M. 2010. How Infection Works - What You Need to Know About Infectious Disease. Washington DC: National Academies Press.

Glo Germ.2014. Glo Germ Powder Safety Data Sheet (All Colors). Utah: Glo Germ Company.

Healthcare-Acquired Infections (HAIs). c2019. Burlington (MA): Massachusetts Health \& Hospital Association - Patient Care Link. [updated 2019; cited 2019 June 1]. https://patientcarelink.org/improvingpatien tcare/healthcare-acquired-infections-hais/.

Home Care. c2019. New Port Richey (FL): C.Diff Foundation. [updated 2019; cited 2019 Sept. 16]. https://cdifffoundation.org/cdiff-infectionhomecare/.

Instructional Design Center [updated 2016 Jan 5; cited 2019 Sept. 16].

Instructional Guide for University Faculty and Teaching Assistants. c2019. DeKalb (IL): Northern Illinois University - Faculty Development and
Mehta, Y., Gupta, A., Todi, S., Myatra, S. N., Samaddar, D. P., Patil, V., Ramasubban, S. 2014. Guidelines for prevention of hospital acquired infections. Indian Journal of Critical Care https://www.niu.edu/facdev/resources/guide/inde x.shtml Medicine, 18(3), 149-163. https://doi.org/10.4103/0972-5229.128705

Most Common Healthcare- Associated Infections: 25 Bacteria, Viruses Causing HAIs. c2019. Chicago (IL): Becker's Healthcare: Becker's Hospital Review. [updated 2014 May 1; cited 2019 Sept. 17].

https://www.beckershospitalreview.com/quality/ most-common-healthcare-associated-infections25-bacteria-viruses-causing-hais.html

What is $C$. diff? c2019. Atlanta (GA): Centers for Disease Control - Clostroiodes difficile (c-diff). [updated 2018 Dec. 17; cited 2019 Sept. 16] https://www.cdc.gov/cdiff/whatis.html\#anchor_1540389114286.

\section{Acknowledgments}

Funding for this project was provided by a Faculty Development Grant and the Biology department at the University of Pittsburgh at Bradford.

\section{About the Authors}

Lexi Green is a Nursing major who will be graduating in 2019 with her Associates degree. She will continue her education to receive her bachelor's degree in an online program in the Fall of 2020.

Susan Wright is a senior at the University of Pittsburgh at Bradford. She is currently pursuing a degree in biology. Her future goal is to work at the National Institute of Environmental Health and Science, examining the effects of the environment on public health.

Sarah E. Ruffell teaches microbiology and has research interested in science. As of 2020, she has begun a new position at the University of Waterloo and would be happy to answer any questions you have at her new emailsruffell@waterloo.ca. 


\section{Appendix A: Sample Data for Vital Signs Flow Sheet}

\section{$\bigoplus$ Vital Signs Flow Sheet}

PATIENT'S NAME: Van Comycin GENDER: MaleFFemale $\quad$ DOB: $3 / 30 / 1984$

\begin{tabular}{|c|c|c|c|c|c|c|c|}
\hline Date: & Temperature: & Pulse: & $\begin{array}{c}\text { Blood } \\
\text { Pressure: }\end{array}$ & Respirations: & $\mathrm{O}_{2}$ Sat: & Pain: & Initials \\
\hline $4 / 09 / 2019$ & $101.4^{\circ} \mathrm{F}$ & $\begin{array}{c}102 \mathrm{beats} \\
\text { per min }\end{array}$ & $104 / 62$ & $\begin{array}{c}22 \text { breaths } \\
\text { per min }\end{array}$ & $98 \%$ & $3 / 10$ & SW \\
\hline & & & & & & & \\
\hline
\end{tabular}

\section{Other Symptoms:}

Patient mentioned: watery diarrhea for 2 days. His abdomen is soft with hyperactive bowel sounds. He mentioned abdominal cramping giving him pain on a level 3 out of 10 . Lungs are clear. Has prolonged tenting when checleing slein vigor.

Figure 2. Key to vital signs sheet. 


\section{Appendix B: Illustrations of Simulation of Bacterial Infection with UV Powder}
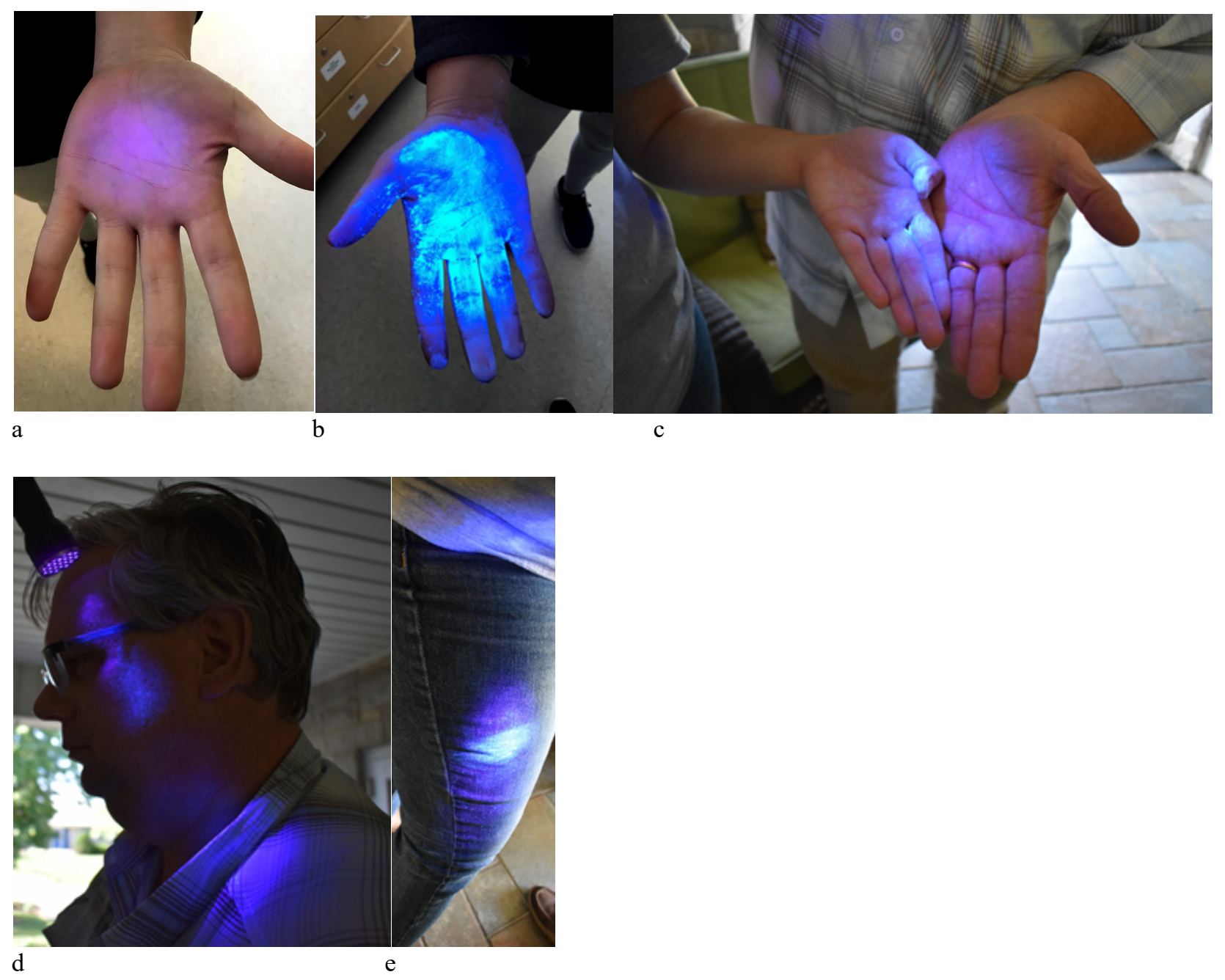

Figure 3. Photograph results of simulation of bacterial infection using UV powder. a) "Uninfected" hand under the UV light. b) "Infected" hand under the UV light. c) Patient's hand next to the doctor's hand. The infection is shown to spread through the transfer of the UV powder via a handshake. d) UV powder on the doctor's glasses to show how the microbes can also be on many of the surfaces we touch daily. e) UV powder on the patient's clothing to provide a visual on how microbes can also spread to many different surfaces that one may encounter. 


\section{Mission, Review Process \& Disclaimer}

The Association for Biology Laboratory Education (ABLE) was founded in 1979 to promote information exchange among university and college educators actively concerned with teaching biology in a laboratory setting. The focus of ABLE is to improve the undergraduate biology laboratory experience by promoting the development and dissemination of interesting, innovative, and reliable laboratory exercises. For more information about ABLE, please visit http://www.ableweb.org/.

Advances in Biology Laboratory Education is the peer-reviewed publication of the conference of the Association for Biology Laboratory Education. Published articles and extended abstracts are evaluated and selected by a committee prior to presentation at the conference, peer-reviewed by participants at the conference, and edited by members of the ABLE Editorial Board. Published abstracts are evaluated and selected by a committee prior to presentation at the conference.

\section{Citing This Article}

Lathrop H, Hull L, Green L, Wright S, Ruffell SE 2020. Demonstrating the spread of bacterial infection within a hospital setting through simulations and role-play. Article $41 \mathrm{In}$ : McMahon K, editor. Advances in biology laboratory education. Volume 41. Publication of the 41st Conference of the Association for Biology Laboratory Education (ABLE). https://doi.org/10.37590/able.v41.art41

Compilation (C) 2020 by the Association for Biology Laboratory Education, ISBN 1-890444-17-0. All rights reserved. No part of this publication may be reproduced, stored in a retrieval system, or transmitted, in any form or by any means, electronic, mechanical, photocopying, recording, or otherwise, without the prior written permission of the copyright owner.

ABLE strongly encourages individuals to use the exercises in this volume in their teaching program. If this exercise is used solely at one's own institution with no intent for profit, it is excluded from the preceding copyright restriction, unless otherwise noted on the copyright notice of the individual chapter in this volume. Proper credit to this publication must be included in your laboratory outline for each use; a sample citation is given above. 